\title{
A meta-analysis approach for assessing the diversity and specificity of belowground root and microbial volatiles
}

\author{
Denis Schenkel ${ }^{1,2}$, Marie C. Lemfack ${ }^{3}$, Birgit Piechulla ${ }^{3}$ and Richard Splivallo ${ }^{1,2 *}$ \\ ${ }^{1}$ Institute for Molecular Biosciences, Goethe University Frankfurt, Frankfurt, Germany, ${ }^{2}$ Integrative Fungal Research Cluster, \\ Frankfurt, Germany, ${ }^{3}$ Institute for Biological Sciences, University of Rostock, Rostock, Germany
}

\section{OPEN ACCESS}

Edited by:

Choong-Min Ryu,

Korea Research Institute of Bioscience and Biotechnology, South Korea

Reviewed by: Huiming Zhang,

Shanghai Center for Plant Stress

Biology - Chinese Academy of Sciences, China Mohamed Farag,

Cairo University, Egypt

*Correspondence:

Richard Splivallo, Institute for Molecular Biosciences,

Goethe University Frankfurt, Max-von-Laue Strasse 9, 60438 Frankfurt, Germany richard.splivallo@a3.epfl.ch

Specialty section:

This article was submitted to Plant Biotic Interactions, a section of the journal Frontiers in Plant Science

Received: 20 July 2015

Accepted: 24 August 2015

Published: 14 September 2015

Citation:

Schenkel D, Lemfack MC, Piechulla B and Splivallo R (2015) A meta-analysis approach for assessing the diversity and specificity of belowground root and microbial volatiles. Front. Plant Sci. 6:707. doi: 10.3389/fpls.2015.00707
Volatile organic compounds are secondary metabolites emitted by all organisms, especially by plants and microbes. Their role as aboveground signals has been established for decades. Recent evidence suggests that they might have a nonnegligible role belowground and might be involved in root-root and root-microbial/pest interactions. Our aim here was to make a comprehensive review of belowground volatile diversity using a meta-analysis approach. At first we synthesized current literature knowledge on plant root volatiles and classified them in terms of chemical diversity. In a second step, relying on the mVOC database of microbial volatiles, we classified volatiles based on their emitters (bacteria vs. fungi) and their specific ecological niche (i.e., rhizosphere, soil). Our results highlight similarities and differences among root and microbial volatiles and also suggest that some might be niche specific. We further explored the possibility that volatiles might be involved in intra- and inter-specific root-root communication and discuss the ecological implications of such scenario. Overall this work synthesizes current knowledge on the belowground volatilome and the potential signaling role of its constituents. It also highlights that the total diversity of belowground volatiles might be orders of magnitude larger that the few hundreds of compounds described to date.

Keywords: microbes, fungi, bacteria, volatiles, diversity, rhizosphere, mycorrhizas, roots

\section{Introduction}

Secondary metabolites are small molecules that are produced by all living organisms. Unlike primary metabolites which are directly involved in regular growth and development, secondary metabolites might be produced only at specific developmental stages or under certain circumstances; hence they might provide a functional readout of cellular state (Patti et al., 2012). Tens of 1000s of secondary metabolites derived from plants and microbes are known to humans as drugs, food additives or flavors, and fragrances; yet, their ecological functions remain poorly understood.

Secondary metabolites indeed play a central role in inter-organismic interactions. In numerous cases volatile and non-volatile secondary metabolites have been implicated in defense 
and communication among organisms. Recently, volatiles have attracted sustained attention, especially in belowground communication, due to their ability to travel further distances than non-volatile metabolites (Rasmann et al., 2005; Wenke et al., 2010; Peñuelas et al., 2014). Because of their potent biological activities on plants, the use of volatiles in agriculture have been suggested as a possible alternative to pesticides (Bitas et al., 2013; Kanchiswamy et al., 2015). A search through literature and databases allows estimating the known structural diversity of volatiles derived from plant flowers - about 1700 volatiles from 991 species (Knudsen et al., 2006; Dunkel et al., 2009) and from microbes, including fungi and bacteria - 1093 volatiles from 491 microbes at the time of this study (Lemfack et al., 2014). Yet considering that $10^{7}-10^{9}$ bacterial species (Schloss and Handelsman, 2004), 1.5 million fungal species (Hawksworth, 2001) and 2,98,000 of plant species (Mora et al., 2011) might exist on earth, the number of volatiles will increase as new species are being characterized and discovered.

In the past 5 years, the ecological role of volatiles in aboveand belowground interactions among plants, fungi, bacteria, and insects has been addressed in a series of comprehensive reviews (Wenke et al., 2010, 2012; Bailly and Weisskopf, 2012; Effmert et al., 2012; Davis et al., 2013; Farag et al., 2013; Audrain et al., 2015; Kanchiswamy et al., 2015; Schmidt et al., 2015). The latest of these reviews (Kanchiswamy et al., 2015) covered literature up to the beginning of 2015. Most recently a further example of belowground volatile based communication has been brought to light for plants and the ectomycorrhizal fungus Laccaria bicolor (Ditengou et al., 2015). Some volatile sesquiterpenoids emitted by the latter fungus were shown to induce root branching in poplar, a host plant which can enter into symbiotic interactions with the fungus, but also in Arabidopsis, a nonhost plant unable of symbiosis with Laccaria. Remarkably not all fungal sesquiterpenoids induced root branching: the volatile (-)thujopsene was implicated in the root morphological change but $\beta$-caryophyllene, another sesquiterpenoid also emitted by maize roots (Rasmann et al., 2005), had no effect on branching. These observations raise questions about the specificity of belowground signals as well as the ability of the target organisms to perceive and react to volatiles.

Soil is actually a highly colonized inhomogeneous substrate. Non-homogeneity is not only reflected in terms of structure and porosity but also in terms of nutritional differences (Schoenholtz et al., 2000). Besides, organisms present in the soil might also provide specific niches for defined microbes, thus exerting a community structuring effect. Belowground community structuring has indeed been observed in numerous cases. A textbook example includes root nodules in legumes which are exclusively colonized by nitrogen fixing rhizobacteria (Gage, 2004). More recent examples are provided by Arabidopsis' root endophytic microbial community made of Proteobacteria, Bacteroidetes, and Actinobacteria (Bulgarelli et al., 2012) and by truffle's fruiting bodies which host bacterial communities clearly distinct from those of the surrounding soil (AntonyBabu et al., 2014). This belowground community structuring might explain why some volatiles could act as successful signaling cues within such communities, however evidence that specific volatiles are emitted in defined habitats/niches is currently limited.

The aim of this paper is to quantify the diversity and explore the specificity of belowground volatiles produced by microbes and plant roots. For this purpose we synthesized existing literature on plant root volatiles and relied on the "mVOC database" of microbial volatiles (Lemfack et al., 2014) to address questions such as: how structurally diverse are plant root and microbial volatiles? Which volatiles are common and specific to microbes and plant roots? Is their emission influenced by microbial phylogeny or habitat; and finally do root volatiles serve as signals for neighboring plants? Overall our aim was to shed more light on belowground volatiles diversity and functions by essentially using a quantitative approach to diversity and by integrating information on the phylogeny and the habitat of the emitters.

\section{Materials and Methods}

\section{Diversity of Plant Root Volatiles}

Volatile organic compounds (VOCs) released by plant roots have been investigated in a limited number of species. Here we gathered information relative to volatile diversity in barely Hordeum vulgare - (29 compounds; Gfeller et al., 2013), the model plant Arabidopsis thaliana (eight compounds; Steeghs et al., 2004), maize - Zea mays (one compound; Rasmann et al., 2005) and the bean Vicia faba (one compound; Babikova et al., 2013). Overall these plant roots emitted 39 volatiles, which have been grouped in Figure 1 based on their biosynthetic origins/chemical classes (i.e., terpenoids, alcohols).

\section{Diversity of Microbial Volatiles}

The diversity of microbial volatiles was investigated using the mVOC database (Lemfack et al., 2014). At the time of this study, the database comprised 1093 volatiles emitted by 135 fungi and 356 bacteria. As for plant roots, volatiles were classified according to chemical classes/biosynthetic origins (Figure 2).

\section{Specificity of Microbial Volatiles Linked to Taxonomy and Ecological Niches}

To understand how specific or common volatiles were in microbes, bacteria and fungi of the mVOC database were classified in taxonomical units either at the phylum or class level. Gaining insight into niche specificity was achieved by classifying the microbes of the mVOC database based on their habitat. Because of our focus on belowground interactions, classification was made in five categories: fungi or bacteria living in the rhizosphere, fungi, or bacteria living in the soil (excluding the rhizosphere), and microbes living in any other habitat (i.e. animals, marine habitats, and microorganisms associated to above-ground plant parts). Classification in specific niches/habitats was based on various data sources which will be shortly included in the mVOC database. Because we were interested in habitat/niche specificity, microbes which were ubiquitous to more than one habitat/niche were excluded from the analysis. 


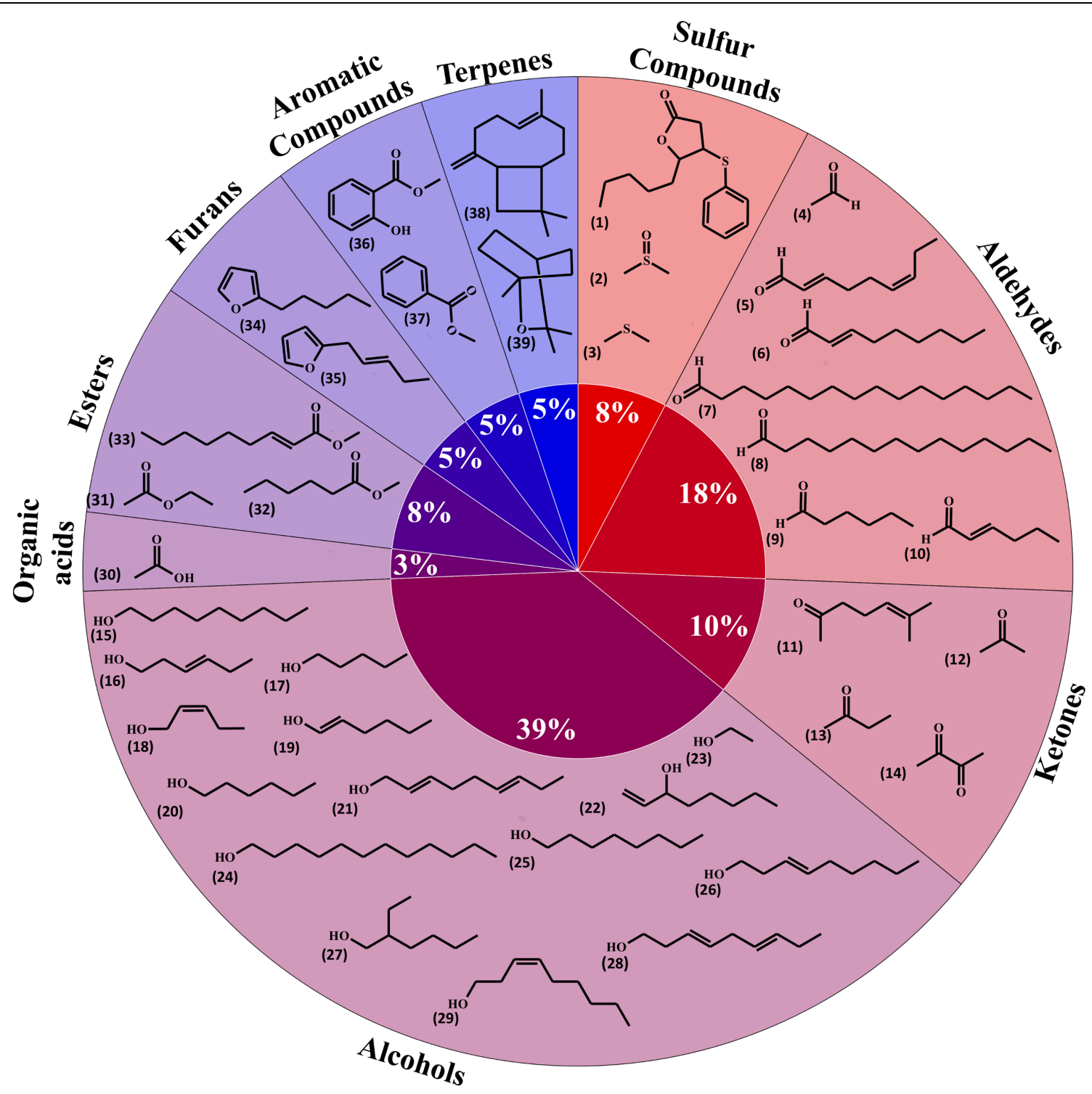

FIGURE 1 | Diversity of plant root volatiles. A total of 39 volatiles released from barley, maize, Arabidopsis or fava bean roots were classified based on their chemical classes and shown here in a pie chart. 5-Pentyl-4-phenylsulfanyloxolan-2-one (1); dimethyl sulfoxide (2); dimethyl sulfide (3); acetaldehyde (4); (2E,6Z)-nona-2,6-dienal (5); (E)-non-2-enal (6); hexadecanal (7); tetradecanal (8); hexanal (9); (2E)-hex-2-enal (10); 6-methylhept-5-en-2-one (11); acetone (12); butan-2-one (13); butane-2,3-dione (14); nonan-1-ol (15); (3E)-hex-3-en-1-ol (16); pentan-1-ol (17); (2Z)-pent-2-en-1-ol (18); (1E)-hex-1-en-1-ol (19); hexan-1-ol (20); nona-2,6-dien-1-ol (21); oct-1-en-3-ol (22); ethanol (23); dodecan-1-ol (24); octan-1-ol (25); (E)-non-3-en-1-ol (26); 2-ethylhexan-1-ol (27); nona-3,6-dien-1-ol (28); (Z)-non-3-en-1-ol (29); acetic acid (30); ethyl acetate (31); methyl hexanoate (32); methyl (E)-non-2-enoate (33); 2-pentylfuran (34); 2-pent-2-enylfuran (35); methyl salicylate (36); methyl-benzoate (37); $\beta$-caryophyllene (38); 1,8-cineole (39).

\section{Effect of Neighboring Plant on Root Development}

The influence of neighboring plants on root development was investigated by compiling data from 18 publications (Mahall and Callaway, 1992; Gersani et al., 2001; Maina et al., 2002; Day et al., 2003; Falik et al., 2003, 2006; Gruntman and Novoplansky, 2004; O’Brien et al., 2005; Dudley and File, 2007; Murphy and Dudley, 2007, 2009; Semchenko et al., 2007, 2014; Broz et al., 2008; Milla et al., 2009; Fang et al., 2013; Schmid et al., 2013a). In all those works root development (biomass or root length depending on the parameter reported) of a plant subjected to neighboring plants was compared to root development of a plant without neighbors. The effects on roots were classified as "increase, decrease, no effect" based on the statistics reported in the papers. Subject plants were grouped either based on genetic relatedness with the interacting plants (as kin, conspecific but not kin, and foreign species) or as monocots and dicots. Cases in which the kinship of individuals of the same species was unspecified were categorized as conspecific. 


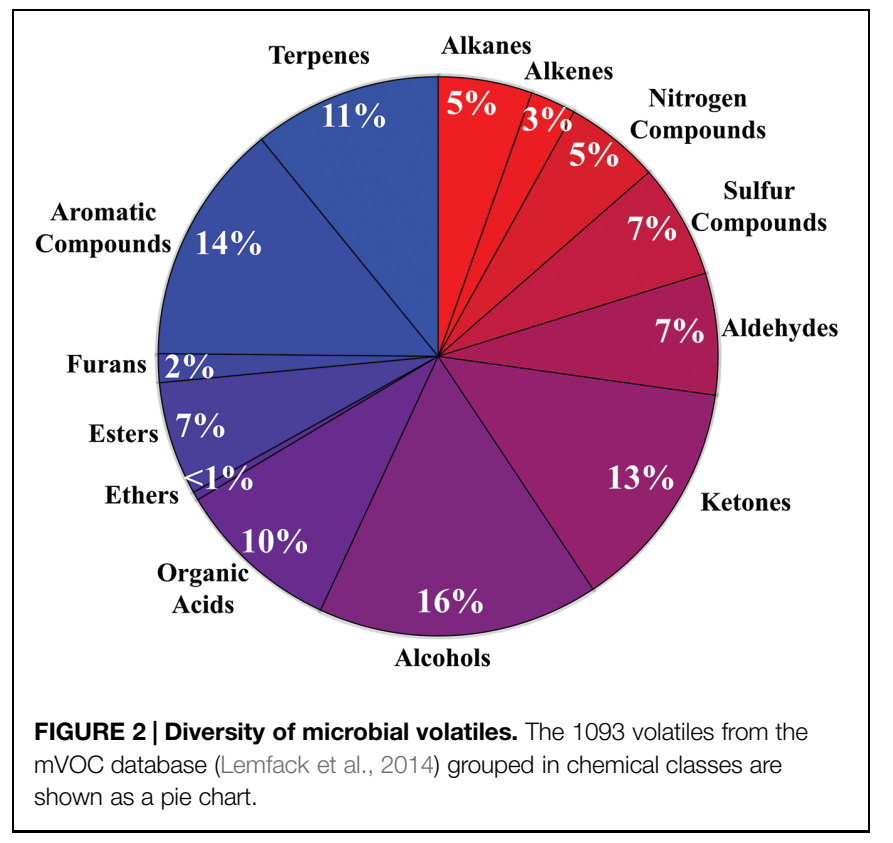

\section{Results}

\section{Diversity of Plant Root and Microbial Volatiles}

Whereas publications investigating volatiles emitted by aboveground plant organs abound, only a few papers have been published on root volatiles, most likely due to the technical difficulties in sampling volatiles in soil matrices. Compiling the information from root volatiles emitted by maize (Rasmann et al., 2005), barley (Gfeller et al., 2013), Arabidopsis thaliana (Steeghs et al., 2004), and the bean Vicia faba (Babikova et al., 2013) revealed an overall diversity of 39 volatiles belonging to nine chemical/biosynthetic groups (Figure 1). With $66 \%$ of all volatiles, alcohols, aldehydes, and ketones represented the major share of root volatiles. The remaining $44 \%$ was composed of minor groups (sulfur compounds, terpenoids, aromatic compounds, furans, esters, and organic acids) each represented by a single or two compounds. By contrast to the scarce information on root volatiles, microbial volatiles have been investigated more thoroughly. An effort to synthesize the large amount of information on microbial volatiles has recently been made through the mVOC database (Lemfack et al., 2014), which also served as the basis of the present study. Here a total of 1093 microbial volatiles from the mVOC database have been grouped according to chemical classes/biosynthetic pathways and the resulting data is presented as a pie chart in Figure 2. Even though some volatiles like ketones, esters, sulfur-containing compounds, and furans appeared with a comparable frequency as in plants roots and microorganisms, the microbial volatilome comprised a greater structural complexity of organic acids, aromatic compounds, and terpenes than plant roots, at least considering the currently available data (Figures 1 and 2). Five groups of microbial volatiles (terpenes, alcohols, ketones, aromatic compounds, and organic acids) represented each $10 \%$ or more of the volatiles, overall accounting for $64 \%$ of the total diversity. Aldehydes, sulfur and nitrogen containing compounds, alkanes, alkenes, furans, ester, and ethers represent minor groups accounting together for almost $37 \%$ of the total diversity.

\section{Which Microbes Produce Plant Root Volatiles?}

A total of 28 plant root volatiles were also produced by microbes. These volatiles included 11 alcohols (dodecan-1-ol; ethanol; 2-ethyl-1-hexanol; hexan-1-ol; 2-hexen-1-ol; 3-hexen1-ol; 1-nonanol; 1-octanol; 1-octen-3-ol; pentanol; 2-penten-1ol), 4 aldehydes (acetaldehyde; hexanal; 2-hexenal; tetradecanal), two aromatic compounds (methyl benzoate; methyl salicylate), two esters (ethyl acetate; methyl hexanoate), one furan (2pentylfuran), four ketones (acetone; butanone; butanedione; 6-methyl-5-hepten-2-one), one organic acid (acetic acid), two sulfur compounds (dimethyl sulfide; sulfinylbismethane), and one terpene ( $\beta$-caryophyllene). Our aim was to understand if these volatiles were preferentially produced by specific bacterial or fungal phyla/classes. For this purpose, microbes emitting plant root volatiles were grouped in phyla and in some cases in classes. The heatmap in Figure 3 represents the percentage of microbes, which are emitters of the plant root volatiles of Figure 1.

In terms of volatile groups, 14 fungal and 22 bacterial phyla emitted plant root volatiles at a rather low frequency $(<10 \%$ as shown from the color scale on the heatmap of Figure 3). Alcohols were the most frequent and were emitted by four of the seven bacterial phyla and by all the fungal phyla. Volatiles belonging to remaining groups were similarly emitted at a low frequency by $50 \%$ of all phyla (fungal and bacterial). Interestingly all volatile groups occurred in at least one fungal and one bacterial phylum. Furans were produced by a fair percentage of fungi belonging to the three fungal phyla considered here whereas it was emitted at low frequency within a single bacterial phylum (Firmicutes, specifically the Bacilli class).

Considering the data in terms of phyla highlighted that members of the Firmicutes and Proteobacteria bacterial phyla and Ascomycetes fungi emitted volatiles belonging to most of the chemical groups. Zooming into bacterial classes revealed that among the Firmicutes, Bacilli were the most frequent emitters of plant root volatiles while among the Proteobacteria, $\beta$-, and $\gamma$-Proteobacteria were the most frequent emitters. Interestingly, acetic acid (the only molecule in the category "organic acid") was produced by about $50 \%$ of all Bacilli and an even higher percentage of Clostridia (the highest percentage with Negativicutes reflects the fact that this class has a single representative).

Overall these results highlight that numerous microbes are capable of emitting the same volatiles as plant roots. They also suggest that some phyla might be better than others at producing these volatiles. Bacteria belonging to the Firmicutes (Bacilli), to the Proteobacteria ( $\beta$ - and $\gamma$-Proteobacteria) and Ascomycetes fungi specifically stand out for their ability to produce a large variety of plant root volatiles.

\section{Common and Specific Volatiles to Plant Roots, Bacteria, and Fungi}

The microbial volatiles of the mVOC database and the plant root volatiles of Figure $\mathbf{1}$ have been presented according to 


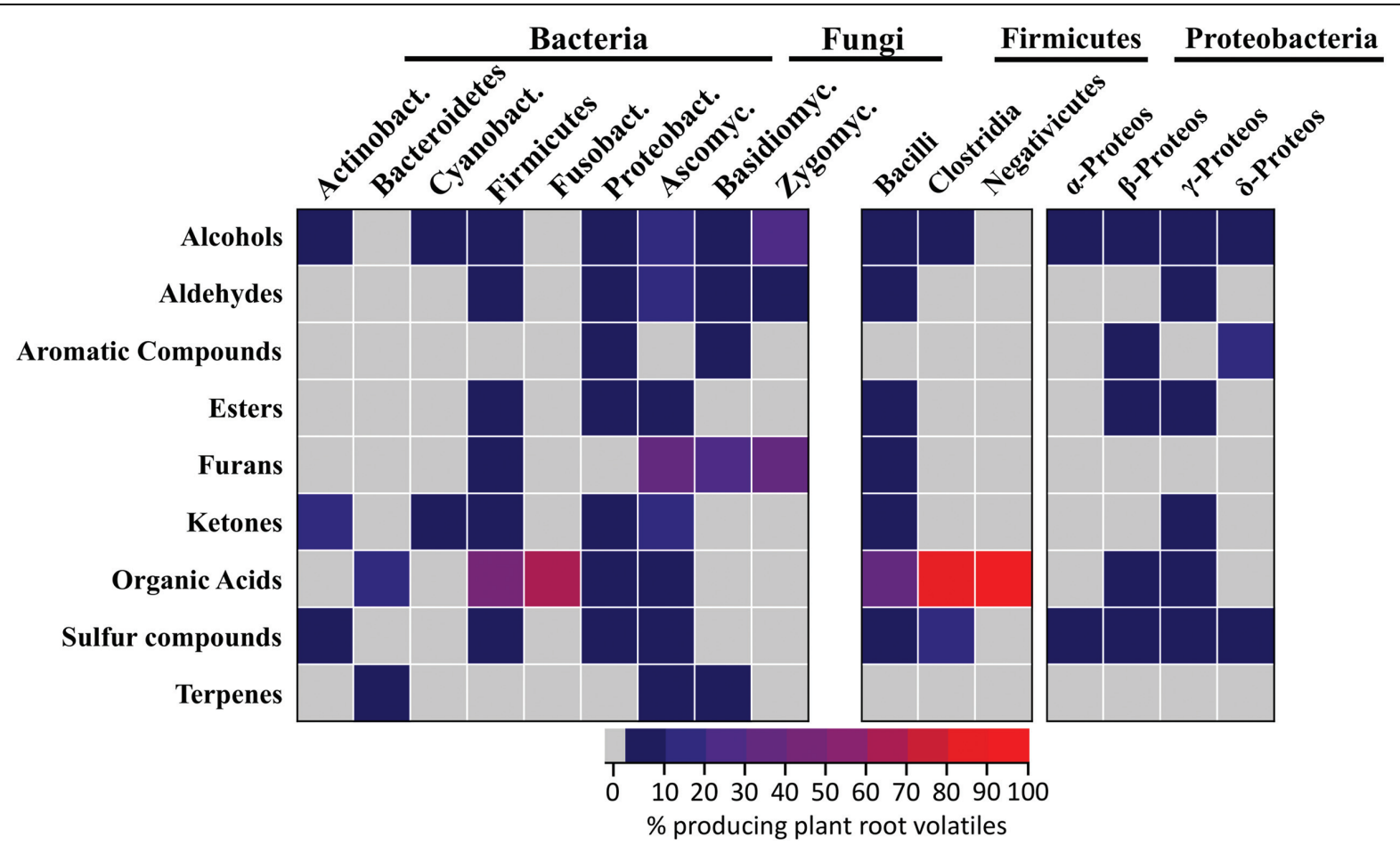

FIGURE 3 | Which microbes produce plant root volatiles? Bacteria and fungi producing the same volatiles as plant roots (Figure 1) were identified from the $m V O C$ database. The results are displayed for the different phyla or classes of bacteria and fungi as a heatmap where each cells represents the percentage of microbes emitting volatiles of Figure 1. Actinobact: Actinobacteria $(n=64)$, Bacteroidetes $(n=44)$, Cyanobact: Cyanobacteria $(n=19)$, Bacilli $(n=11)$, Clostridia $(n=10)$, Negativicutes $(n=1)$, Fusobact: Fusobacteria $(n=3), \alpha$-Proteos: $\alpha$-Proteobacteria $(n=25), \beta$-Proteos: $\beta$-Proteobacteria $(n=43), \gamma$-Proteos:

$\gamma$-Proteobacteria $(n=62), \delta$-Proteos: $\delta$-Proteobacteria $(n=17)$, Ascomyc: Ascomycota $(n=104)$, Basidiomyc: Basidiomycota $(n=28)$, Zygomyc: Zygomycota $(n=3)$.

the potential origin/habitat of their emitters. These origins have been regrouped here in five categories as plant roots (39 volatiles), rhizosphere fungi (261 volatiles), rhizosphere bacteria (209 volatiles), soil fungi (187 volatiles), soil bacteria (483 volatiles). The data is presented as a Venn diagram highlighting the number of specific and common volatiles among groups (Figure 3).

A total of 853 volatiles were emitted by plant roots and belowground microbes. Considering the five groups defined here, all groups shared eight volatiles; however, the majority of volatiles were unique to distinct origins/habitats. For example, of the 39 volatiles produced by plant roots (Figure 1), 12 (or 31\%) were solely produced by roots and not by any other microbes. Depending on their habitat fungi produced 145 (rhizosphere) and 96 (soil) unique volatiles not shared by any other groups; by contrast soil and rhizosphere fungi had 61 volatiles in common. The same argument can be made for bacteria, which produced 76 (rhizosphere) and 297 (soil) unique volatiles, and shared 126 of them.

Overall this data exemplifies the specificity but also the extent of the overlap in volatile signals emitted by plant roots and microbes. It highlights the existence of a core volatilome for bacteria and plant roots but also the fact that a high proportion of volatiles are specific to organisms in defined habitats.

\section{Are Microbial Volatiles Niche Specific?}

Fungi and bacteria from the mVOC database were regrouped according to their lifestyle/habitat. Similarly to Figure 4 three categories were considered in relation to possible interactions with plants: organisms typically found in the soil (S), microbes associated with the rhizosphere (R) and organisms which did not fall in those two categories (N) (i.e., either associated to above plant organs or with animals). Only volatiles occurring in at least 10 microbes are shown here. Values in the heatmap represent the percentage of microbes emitting a specific volatile in each category.

In terms of chemical classes/groups, numerous terpenes, aromatic compounds, nitrogen, and sulfur containing compounds, alkanes and alkenes were predominantly produced by bacteria compared to fungi. Some volatiles such as nitrogen containing compounds were actually almost exclusively produced by bacteria. By contrast no volatiles were exclusively produced by fungi. In most cases habitat specificity (i.e., soil, rhizosphere) seemed to have little influence on volatiles patterns. Volatiles belonging to a few groups were, however, predominantly produced by rhizosphere (R) organisms (in opposite to soil (S) and "other" (N) organisms). This was the case for example in fungi for alcohols, sulfur compounds, some aromatic compounds (i.e., 2-phenylethanol) and some ketones (i.e., octan-3-one). Similarly in bacteria nitrogen containing 


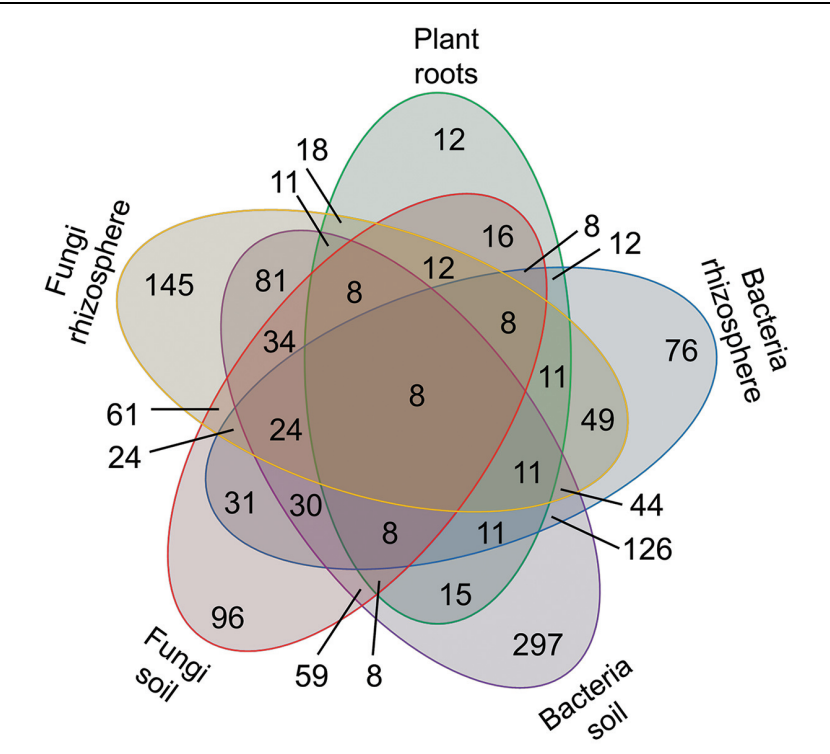

FIGURE 4 | Number of volatiles specific and common to plant roots, bacteria, and fungi. A total of 853 volatiles emitted by plant roots (Figure 1) and belowground microbes were grouped based on their source/habitat in the five categories and are displayed in a Venn diagram. The numbers of volatiles specific or shared among categories are displayed here.

compounds production seemed slightly higher in rhizosphere organisms.

Plant root volatiles shown in bold were marked with an asterisk in Figure 5. With the exception of 1-octen-3-ol, most of these volatiles were emitted by microbes in most/all categories. Nevertheless it is noteworthy that six of the eight plant root volatiles shown here [2-pentylfuran; dimethyl sulfide (syn. (methylsulfanyl)methane]; ethyl acetate; acetone; ethanol; 1-octen-3-ol) were emitted by a comparatively higher percentage of rhizospheric fungi compared to fungi and bacteria colonizing different habitats.

These results demonstrate marked differences in terms of volatile production patterns among bacteria and fungi. This suggests that bacteria might be capable of synthesizing structurally more diverse volatiles than fungi. They also indicate that microbes belonging to specific niches/habitats, especially to the rhizosphere, might preferentially produce volatile signals, including many of the volatiles also emitted by plant roots.

\section{Could Root Volatiles be Perceived by Neighboring Plants?}

There is a mounting body of evidence that neighboring plants can communicate with each other through their roots (Dudley and File, 2007; Bhatt et al., 2011; Fang et al., 2013; Schmid et al., 2013b). Obvious signals for such communication might be volatile molecules. Additionally, volatile emission patterns of aboveground plant organs were shown to be dependent on genetic relatedness. For the sake of clarity, kin plants by definition share the same parents/ancestors, as opposed to conspecific plants which, besides belonging to one species, do not have common parents/ancestors. Recently volatile profiles of kins were shown to be more similar to each other than those of plants without kinship (conspecific plants) (Karban et al., 2014). This led us to question whether plant roots react differently to neighboring plants based on their genetic relatedness (i.e., kins, conspecific but not kins, or foreign (different species) see cartoon of Figure 6). Furthermore we also questioned if differences existed among monocots and dicots.

To answer these questions we gathered publications, which compared root development of one plant with a neighbor to a single plant without neighbor. A total of 30 observations from 18 publications were taken into account and their outcomes have been synthesized in Figure 6.

Comparing kins to conspecifics revealed that the roots of more than $50 \%$ of kins were unaffected by their neighbors compared to only $21 \%$ for conspecifics. When an effect was observed, this predominantly corresponded to an increased root biomass for both categories. The opposite was true for plants subjected to a foreign neighbor. These predominantly ( $43 \%$ of all observations) reacted to the neighbor by decreasing or shortening their roots. Patterns were less obvious with monocots and dicots. Indeed the number of cases in which roots were either affected (increase or decrease in biomass/root length) or unaffected were comparable.

Overall these results highlight that among kin, foreign and conspecific plants, roots of kins are the less likely to be influenced by a neighbor. They also exemplify that plants sharing the same genotype (kins and conspecifics) might predominantly react to each other by increasing their root biomass/root length, while plants with a foreign neighbor might commonly decrease their root biomass.

\section{Discussion}

During the past decade VOCs have gained recognition as essential signals in inter-organismic interactions. Especially belowground volatiles might convey information among plant roots, microbes, and insects. The diversity of volatiles and effects on their target organisms have been recently synthesized in a series of comprehensive reviews (Wenke et al., 2010, 2012; Bailly and Weisskopf, 2012; Effmert et al., 2012; Davis et al., 2013; Kanchiswamy et al., 2015). Our aim here was to bring this synthesis one step further by using a quantitative meta-analysis approach and integrating data about phylogeny and potential habitat of the emitters.

\section{Diversity of Belowground Volatiles}

Adding up volatiles emitted by plant roots to volatiles emitted by soil/root microbes brings the total diversity of belowground volatiles to 853 . Considering the scarce information on plant root volatiles (only a few existing publications), and the huge unexplored diversity of soil microbes, the overall diversity of belowground volatiles might be orders of magnitudes higher than the few 100s of compounds described to date.

A note of caution should nevertheless be used when estimating diversity from literature data, since most studies describing volatiles from microbes or plant roots have been conducted under laboratory (and sometimes axenic) conditions. Indeed it 


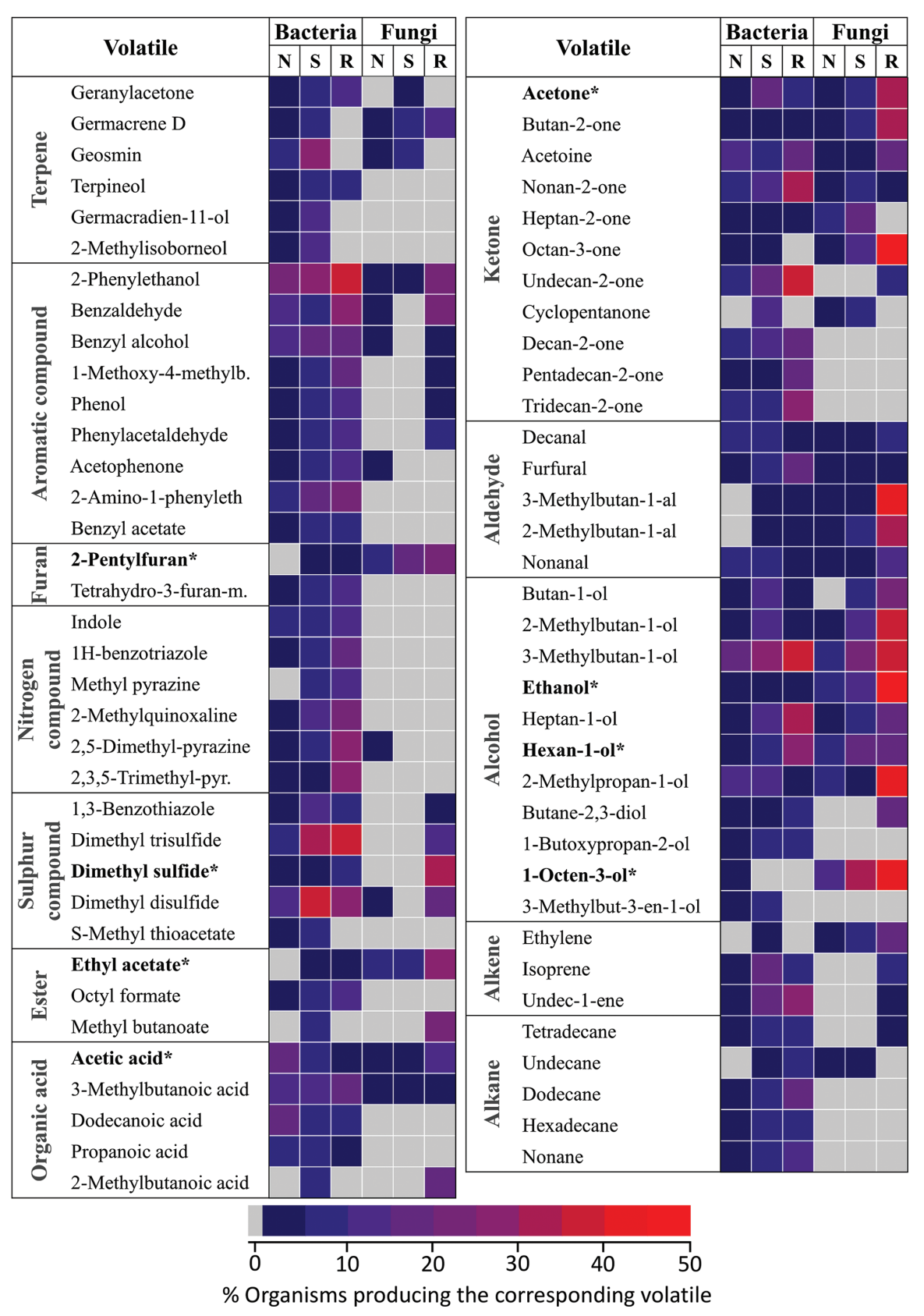

FIGURE 5 | Niche specificity of bacterial and fungal volatiles. Volatiles emitted by at least 10 different organisms from the mVOC database are shown. Microbes were classified based on their lifestyle/habitat as "soil" (120 bacteria, 36 fungi), "rhizosphere" (rhizo: 43 bacteria, 26 fungi) and "other" (157 bacteria, 64 fungi). The color code shows the frequency of each volatile in each group. *These volatiles are also emitted by plant roots.

is well known that media composition, culture conditions or interacting organisms might influence secondary metabolism (Blom et al., 2011; Brakhage and Schroeckh, 2011). Hence if the presence of one volatile in the mVOC database reflects the ability of specific organisms to produce that volatile, its absence does not exclude that it might be produced under natural conditions. The reverse is certainly also true. Overall estimating the total diversity of belowground volatiles will require isolating and characterizing more microbes/plant roots but also analyzing full soil communities under both laboratory and natural conditions. It should be highlighted that profiling volatiles from soil is much more complicated than from any other system. Indeed soil is a highly complex matrix which requires the most advanced instrumentation in terms of resolution and sensitivity (i.e., high resolution MS or proton transfer MS) as well as powerful data processing for harnessing the complexity of its volatilome (Peñuelas et al., 2014; Mancuso et al., 2015). 

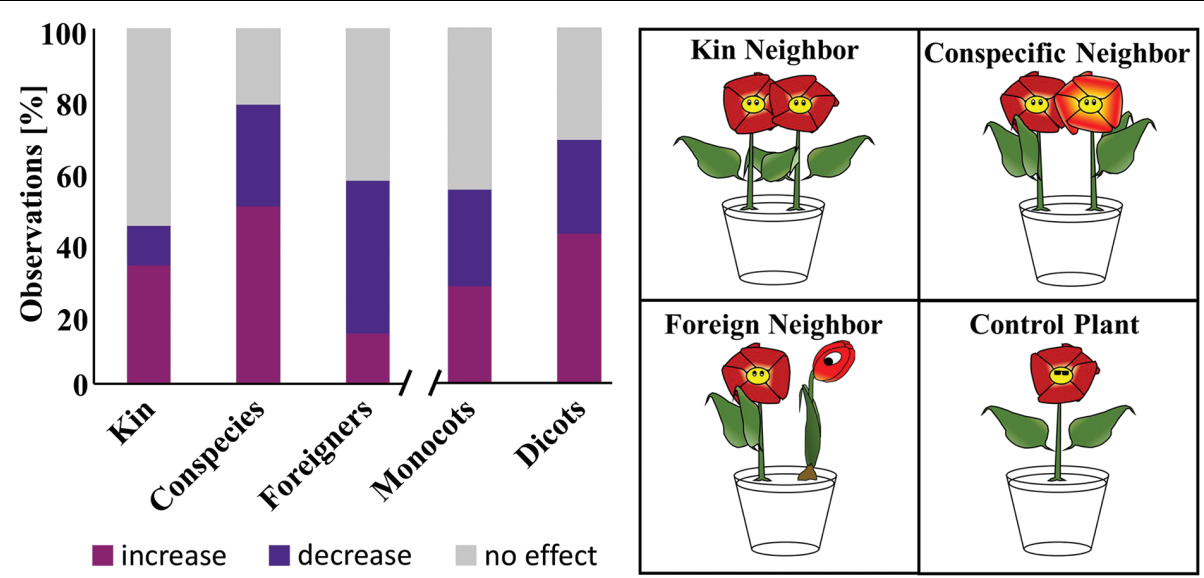

FIGURE 6 | Influence of a neighboring plant on root development. Data presented in a bar chart summarizes the observations of several studies which compared root development of a plant with and without neighbor. The cartoon on the right illustrates the interactions considered here. "Increase, decrease, no effect" refer to the root biomass of a plant with neighbor compared to the one without. Kin: 20 observations in which interacting plants are kins. Conspecies: 27 observations where tested plants are from the same species (but not kins). Foreign: 45 observations where interacting plants belong to different species. Monocots: 28 observations involving monocotyledonous plants. Dicot: 64 observations involving dicotyledonous plants.

\section{Differences and Similarities in Volatile Profiles of Roots, Bacteria, and Fungi}

Our analysis highlighted similarities and differences among plant root and belowground microbial volatiles. In terms of similarities, numerous microbes were capable of emitting the same volatiles as plant roots; however, bacteria belonging to the Firmicutes (Bacilli), to the Proteobacteria ( $\beta$ - and $\gamma$-Proteobacteria) and Ascomycete fungi especially distinguished themselves in this regard. Interestingly Firmicutes and $\beta$ - and $\gamma$-Proteobacteria tend to be dominant root endophytes in rice and sugarcane (Fischer et al., 2011; Sessitsch et al., 2012). Ascomycete fungi also include numerous members which live in close association with plant roots (i.e., truffles forming ectomycorrhizas; Martin et al., 2010). It is therefore tempting to speculate that resemblance in terms of volatile profiles might translate into closer associations between microbes and plant roots. Testing this hypothesis will require characterizing the volatile profiles of numerous plant roots and microbial strains under natural conditions.

\section{How Specific are Belowground Volatile Signals?}

Determining how specific volatile signals might be in terms of interactions requires understanding the nature of the interaction and also the habitat in which it takes place. In terms of molecules, terpenoids are not only important volatiles for floral scent (Knudsen et al., 1993, 2006; Pichersky and Gershenzon, 2002), but as illustrated by $\beta$-caryophyllene in maize, they might serve as an alarm signal upon attack by root pests (Rasmann et al., 2005). This volatile is also emitted by a bacterium belonging to the Bacteroidetes phylum and by some fungi (Figure 3; Lemfack et al., 2014). It has additionally been reported from the fungus Fusarium oxysporum colonized by ectosymbiotic bacteria, and it is responsible of the growth promoting effect observed in lettuce colonized by the latter fungus (Minerdi et al., 2011). This example illustrates that one volatile might be produced by numerous organisms to a different end. Another terpenoid, geosmin, which is produced by numerous microbes (Lemfack et al., 2014) was also recently reported from beet roots (Beta vulgaris sp. vulgaris) (Freidig and Goldman, 2014). We had originally not included this compound in our list of plant root volatiles because they were suspicions that it might not have been produced by beet root itself but by microbes colonizing beet roots tissues; however, the data presented by Freidig and Goldman (2014) suggests that this might be otherwise. This highlights that characterizing the volatile profiles of existing plant roots might greatly increase the diversity of plant root volatiles.

Sulfur containing volatiles are also important signals in plantmicrobe interactions. Indeed it has recently been demonstrated that dimethyl disulfide produced by Bacillus bacteria naturally colonizing tobacco roots promoted plant growth by enhancing sulfur assimilation (Meldau et al., 2013). Our data highlights that this volatile is predominantly produced by bacteria (essentially soil bacteria) and to a lesser extent by fungi (Figure 5). A tempting interpretation might be that numerous soil bacteria might use this volatile for plant growth promotion. Other bacterial volatiles might also serve this purpose, indeed 2,3butanediol promotes plant growth in Arabidopsis (Ryu et al., 2003). Nevertheless the overall effect of microbial volatiles on plant growth depends on the total volatile blend and cultural conditions of the microbes (Blom et al., 2011; Peñuelas et al., 2014). Therefore understanding the specificity of a signal also requires characterizing the context in which it is emitted as well as the bioactivity of the total volatile blend.

Eight carbon containing volatiles are characteristic of fungi, and its major representative, 1-octen-3-ol is indeed responsible of the typical fungal smell perceived by humans (Wnuk et al., 1983; Mosandl et al., 1986). Our data indicates that 1-octen-3ol and octan-3-one are predominantly produced by rhizospheric fungi (Figure 5). Since numerous of these fungi live in 
symbiotic association with plant roots (i.e., truffles), eight carboncontaining volatiles might serve as symbiotic signals to a potential host plant. In terms of biological activity high concentrations of these volatiles have been shown to inhibit seed germination and seedling development in Arabidopsis and Cistus incanus, a host plant to truffles (Splivallo et al., 2007; Hung et al., 2014). Nevertheless lower concentrations of 1-octen-3-ol was shown to induce plant defense genes in Arabidopsis (Kishimoto et al., 2007). These volatile signals might therefore modulate the hostplant fitness, however, how effective this modulation might be in nature remains to be investigated.

Another group of potential signaling molecules are nitrogencontaining volatiles. Interestingly, these volatiles seem essentially produced by bacteria but not by fungi. In relation to their habitat, rhizosphere bacteria were the best producers of these volatiles (Figure 5). Since these bacteria include the Rhizobium genus, members of which are able to fix atmospheric nitrogen and hence literally serve as natural fertilizers for legumes when colonizing their roots (Gage, 2004), it can be speculated that nitrogen-containing volatiles are involved in signaling between Rhizobium and legumes. As in the case of dimethyl disulfide (Meldau et al., 2013), nitrogen-containing volatiles might be directly assimilated by legumes for nutritional purposes, however, they might serve other purposes as well. Demonstrating their exact role as signaling agents will first require deciphering their biosynthesis.

The examples above illustrate how specific or unspecific belowground volatile signals might be. The various ecological roles highlighted here and, in some cases, the ability of different organisms to emit the same signals, suggest the existence of complex volatile-based interaction networks. Demonstrating their specificity will require characterizing full networks of interacting organisms but also concentrationsactivity ratios as well as the persistence of volatile signals in soil.

\section{Could Root Volatiles be Perceived by Neighboring Plants?}

Plants are able to communicate belowground with their neighbors through some unknown signals (Dudley and File, 2007; Bhatt et al., 2011; Fang et al., 2013; Schmid et al., 2013b). Genetic relatedness has recently emerged as an important factor governing belowground root-root interactions. For example roots of rice plants belonging to the same genotype were show to grow toward each other whereas those of different genotypes seem to avoid each other (Fang et al., 2013). Another study involving Cakile edentula plants illustrated that plant root allocation is influenced by kinship; indeed the authors observed lower root allocation in kin pairs than stranger pairs (Bhatt et al., 2011). The nature of the signals involved in root-root communication has not yet been fully identified, however, root exudates have been recently suggested as possible candidates (Semchenko et al., 2014). Because volatiles can essentially diffuse further in the soil than root exudates, they might also act as signaling agents in root-root communication. We explored this possibility relying here on indirect evidence. Indeed volatile emission patterns of aboveground plant organs were recently shown to be dependent on kinship, with volatile profiles of kins being more similar to each other than those of plants without kinship (but of the same species) (Karban et al., 2014). Our data demonstrates that how plants respond in terms of root biomass/structure to the presence of a neighboring plant is actually influenced to a certain extent by kinship and genetic relatedness (Figure 6). Taken as a whole this suggests that volatile signals might indeed be involved in belowground rootroot communications. Demonstrating their exact involvement will require profiling root volatiles as a function of genetic relatedness, identifying the signaling agents, and demonstrating their activity.

\section{Conclusion}

The past decade has seen an increasing interest in belowground volatile-based communication among organisms (Kai et al., 2009; Wenke et al., 2010, 2012; Bailly and Weisskopf, 2012; Piechulla and Degenhardt, 2014). Because of the high heterogeneity and large organismic diversity present in the soil, and the potentially humongous diversity of belowground volatiles, it is essential to apply a holistic approach to understand diversity. Such an attempt has been made here essentially relying on the recently published $\mathrm{mVOC}$ database of microbial volatiles (Lemfack et al., 2014) and on a limited number of papers describing plant root volatiles. Although our analysis highlights interesting patterns in belowground volatile diversity and distribution, it also cries out for more data. Essentially we might be looking at the tip of the iceberg and estimating total belowground volatile diversity will require characterizing both the emitters and their full volatile spectra. This will be a major challenge considering the huge number of undescribed soil microbes.

\section{Author Contributions}

DS and RS wrote the manuscript with input from the other co-authors. ML and BP acquired the data. DS and RS further analyzed and categorized the data and DS created the illustrations. All authors approved the final version of the manuscript.

\section{Funding}

Financial support was provided for DS and RS by the LOEWE research funding program of the government of Hessen, in the framework of the Integrative Fungal Research Cluster (IPF). BP thanks the DFG for financial support.

\section{Acknowledgment}

We are thankful to Mrs. Maryam Vahdatzadeh and Mr. Mohammed Sherif for their support in processing the raw data from the mVOC database. 


\section{References}

Antony-Babu, S., Deveau, A., Van Nostrand, J. D., Zhou, J., Le Tacon, F., Robin, C., et al. (2014). Black truffle-associated bacterial communities during the development and maturation of Tuber melanosporum ascocarps and putative functional roles. Environ. Microbiol. 16, 2831-2847. doi: 10.1111/1462-2920. 12294

Audrain, B., Farag, M. A., Ryu, C.-M., and Ghigo, J.-M. (2015). Role of bacterial volatile compounds in bacterial biology. FEMS Microbiol. Rev. 39, 222-233. doi: 10.1093/femsre/fuu013

Babikova, Z., Gilbert, L., Bruce, T. J. A., Birkett, M., Caulfield, J. C., Woodcock, C., et al. (2013). Underground signals carried through common mycelial networks warn neighbouring plants of aphid attack. Ecol. Lett. 16, 835-843. doi: 10.1111/ele.12115

Bailly, A., and Weisskopf, L. (2012). The modulating effect of bacterial volatiles on plant growth: current knowledge and future challenges. Plant Signal. Behav. 7, 79-85. doi: 10.4161/psb.7.1.18418

Bhatt, M. V., Khandelwal, A., and Dudley, S. A. (2011). Kin recognition, not competitive interactions, predicts root allocation in young Cakile edentula seedling pairs. New Phytol. 189, 1135-1142. doi: 10.1111/j.14698137.2010.03548.x

Bitas, V., Kim, H.-S., Bennett, J. W., and Kang, S. (2013). Sniffing on microbes: diverse roles of microbial volatile organic compounds in plant health. Mol. Plant Microbe Interact. 26, 835-843. doi: 10.1094/MPMI-10-120249-CR

Blom, D., Fabbri, C., Connor, E. C., Schiestl, F. P., Klauser, D. R., Boller, T., et al. (2011). Production of plant growth modulating volatiles is widespread among rhizosphere bacteria and strongly depends on culture conditions. Environ. Microbiol. 13, 3047-3058. doi: 10.1111/j.1462-2920.2011.02582.x

Brakhage, A. A., and Schroeckh, V. (2011). Fungal secondary metabolites strategies to activate silent gene clusters. Fungal. Genet. Biol. 48, 15-22. doi 10.1016/j.fgb.2010.04.004

Broz, A. K., Manter, D. K., Callaway, R. M., Paschke, M. W., and Vivanco, J. M. (2008). A molecular approach to understanding plant-plant interactions in the context of invasion biology. Funct. Plant Biol. 35, 1123-1134. doi 10.1071/FP08155

Bulgarelli, D., Rott, M., Schlaeppi, K., Ver Loren van Themaat, E. Ahmadinejad, N., Assenza, F., et al. (2012). Revealing structure and assembly cues for Arabidopsis root-inhabiting bacterial microbiota. Nature 488, 91-95. doi: 10.1038/nature11336

Davis, T. S., Crippen, T. L., Hofstetter, R. W., and Tomberlin, J. K. (2013). Microbial volatile emissions as insect semiochemicals. J. Chem. Ecol. 39, 840-859. doi: 10.1007/s10886-013-0306-Z

Day, K. J., John, E. A., and Hutchings, M. J. (2003). The effects of spatially heterogeneous nutrient supply on yield, intensity of competition and root placement patterns in Briza media and Festuca ovina. Funct. Ecol. 17, 454-463. doi: 10.1046/j.1365-2435.2003.00758.x

Ditengou, F. A., Müller, A., Rosenkranz, M., Felten, J., Lasok, H., van Doorn, M. M., et al. (2015). Volatile signalling by sesquiterpenes from ectomycorrhizal fungi reprogrammes root architecture. Nat. Commun. 6, 6279. doi: $10.1038 /$ ncomms7279

Dudley, S. A., and File, A. L. (2007). Kin recognition in an annual plant. Biol. Lett. 3, 435-438. doi: 10.1098/rsbl.2007.0232

Dunkel, M., Schmidt, U., Struck, S., Berger, L., Gruening, B., Hossbach, J., et al. (2009). SuperScent-a database of flavors and scents. Nucleic Acids Res. 37, D291-D294. doi: 10.1093/nar/gkn695

Effmert, U., Kalderás, J., Warnke, R., and Piechulla, B. (2012). Volatile mediated interactions between bacteria and fungi in the soil. J. Chem. Ecol. 38, 665-703. doi: 10.1007/s10886-012-0135-135

Falik, O., de Kroon, H., and Novoplansky, A. (2006). Physiologically-mediated self/non-self root discrimination in trifolium repens has mixed effects on plant performance. Plant Signal. Behav. 1, 116-121. doi: 10.4161/psb.1.3.2639

Falik, O., Reides, P., Gersani, M., and Novoplansky, A. (2003). Self/nonself discrimination in roots. J. Ecol. 91, 525-531. doi: 10.1046/j.13652745.2003.00795.x

Fang, S., Clark, R. T., Zheng, Y., Iyer-Pascuzzi, A. S., Weitz, J. S., Kochian, L. V., et al. (2013). Genotypic recognition and spatial responses by rice roots. Proc. Natl. Acad. Sci. U.S.A. 110, 2670-2675. doi: 10.1073/pnas. 1222821110
Farag, M. A., Zhang, H., and Ryu, C.-M. (2013). Dynamic chemical communication between plants and bacteria through airborne signals: induced resistance by bacterial volatiles. J. Chem. Ecol. 39, 1007-1018. doi: 10.1007/s10886013-0317-319

Fischer, D., Pfitzner, B., Schmid, M., Simões-Araújo, J. L., Reis, V. M., Pereira, W., et al. (2011). Molecular characterisation of the diazotrophic bacterial community in uninoculated and inoculated field-grown sugarcane (Saccharum sp.). Plant Soil 356, 83-99. doi: 10.1007/s11104-011-0812-810

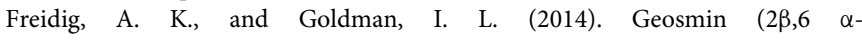
Dimethylbicyclo[4.4.0] decan-1 $\beta$-ol) production associated with Beta vulgaris ssp. vulgaris is cultivar specific. J. Agric. Food Chem. 62, 2031-2036. doi: $10.1021 /$ jf 4047336

Gage, D. J. (2004). Infection and invasion of roots by symbiotic, nitrogen-fixing rhizobia during nodulation of temperate legumes. Microbiol. Mol. Biol. Rev. 68, 280-300. doi: 10.1128/MMBR.68.2.280-300.2004

Gersani, M., Brown, J. S., O’Brien, E. E., Maina, G. M., and Abramsky, Z. (2001). Tragedy of the commons as a result of root competition. J. Ecol. 89, 660-669. doi: 10.1046/j.0022-0477.2001.00609.x

Gfeller, A., Laloux, M., Barsics, F., Kati, D. E., Haubruge, E., Jardin, P. et al. (2013). Characterization of volatile organic compounds emitted by barley (Hordeum vulgare L.) roots and their attractiveness to wireworms. J. Chem. Ecol. 39, 1129-1139. doi: 10.1007/s10886-013-0302-303

Gruntman, M., and Novoplansky, A. (2004). Physiologically mediated self/nonself discrimination in roots. Proc. Natl. Acad. Sci. U.S.A. 101, 3863-3867. doi: $10.1073 /$ pnas.0306604101

Hawksworth, D. L. (2001). The magnitude of fungal diversity: the 1.5 million species estimate revisited. Mycol. Res. 105, 1422-1432. doi: 10.1017/S0953756201004725

Hung, R., Lee, S., Rodriguez-Saona, C., and Bennett, J. W. (2014). Common gas phase molecules from fungi affect seed germination and plant health in Arabidopsis thaliana. AMB Express 4, 53. doi: 10.1186/s13568-0140053-58

Kai, M., Haustein, M., Molina, F., Petri, A., Scholz, B., and Piechulla, B. (2009). Bacterial volatiles and their action potential. Appl. Microbiol. Biotechnol. 81, 1001-1012. doi: 10.1007/s00253-008-1760-1763

Kanchiswamy, C. N., Malnoy, M., and Maffei, M. E. (2015). Chemical diversity of microbial volatiles and their potential for plant growth and productivity. Front. Plant. Sci. 6:151. doi: 10.3389/fpls.2015.00151

Karban, R., Wetzel, W. C., Shiojiri, K., Ishizaki, S., Ramirez, S. R., and Blande, J. D. (2014). Deciphering the language of plant communication: volatile chemotypes of sagebrush. New Phytol. 204, 380-385. doi: 10.1111/nph.12887

Kishimoto, K., Matsui, K., Ozawa, R., and Takabayashi, J. (2007). Volatile 1-octen3-ol induces a defensive response in Arabidopsis thaliana. J. Gen. Plant Pathol. 73, 35-37. doi: 10.1007/s10327-006-0314-318

Knudsen, J. T., Eriksson, R., Gershenzon, J., and Ståhl, B. (2006). Diversity and distribution of floral scent. Bot. Rev. 72, 1-120. doi: 10.1663/00068101(2006)72[1:DADOFS]2.0.CO;2

Knudsen, J. T., Tollsten, L., and Bergström, L. G. (1993). Floral scents-a checklist of volatile compounds isolated by head-space techniques. Phytochemistry 33, 253-280. doi: 10.1016/0031-9422(93)85502-I

Lemfack, M. C., Nickel, J., Dunkel, M., Preissner, R., and Piechulla, B. (2014). mVOC: a database of microbial volatiles. Nucleic Acids Res. 42, D744-D748. doi: 10.1093/nar/gkt1250

Mahall, B. E., and Callaway, R. M. (1992). Root communication mechanisms and intracommunity distributions of two mojave desert shrubs. Ecology 73, 2145-2151. doi: 10.2307/1941462

Maina, G. G., Brown, J. S., and Gersani, M. (2002). Intra-plant versus Inter-plant Root Competition in Beans: avoidance, resource matching or tragedy of the commons. Plant Ecol. 160, 235-247. doi: 10.1023/A:1015822003011

Mancuso, S., Taiti, C., Bazihizina, N., Costa, C., Menesatti, P., Giagnoni, L., et al. (2015). Soil volatile analysis by proton transfer reaction-time of flight mass spectrometry (PTR-TOF-MS). Appl. Soil Ecol. 86, 182-191. doi: 10.1016/j.apsoil.2014.10.018

Martin, F., Kohler, A., Murat, C., Balestrini, R., Coutinho, P. M., Jaillon, O., et al. (2010). Périgord black truffle genome uncovers evolutionary origins and mechanisms of symbiosis. Nature 464, 1033-1038. doi: 10.1038/nature08867

Meldau, D. G., Meldau, S., Hoang, L. H., Underberg, S., Wünsche, H., and Baldwin, I. T. (2013). Dimethyl disulfide produced by the naturally associated bacterium 
bacillus sp B55 promotes Nicotiana attenuata growth by enhancing sulfur nutrition. Plant Cell 25, 2731-2747. doi: 10.1105/tpc.113.114744

Milla, R., Forero, D. M., Escudero, A., and Iriondo, J. M. (2009). Growing with siblings: a common ground for cooperation or for fiercer competition among plants? Proc. R. Soc. Lond. B Biol. Sci. 276, 2531-2540. doi: 10.1098/rspb.2009.0369

Minerdi, D., Bossi, S., Maffei, M. E., Gullino, M. L., and Garibaldi, A. (2011). Fusarium oxysporum and its bacterial consortium promote lettuce growth and expansin A5 gene expression through microbial volatile organic compound (MVOC) emission. FEMS Microbiol. Ecol. 76, 342-351. doi: 10.1111/j.15746941.2011.01051.x

Mora, C., Tittensor, D. P., Adl, S., Simpson, A. G. B., and Worm, B. (2011). How many species are there on Earth and in the Ocean? PLoS Biol. 9:e1001127. doi: 10.1371/journal.pbio.1001127

Mosandl, A., Heusinger, G., and Gessner, M. (1986). Analytical and sensory differentiation of 1-octen-3-ol enantiomers. J. Agric. Food Chem. 34, 119-122. doi: $10.1021 /$ jf00067a033

Murphy, G. P., and Dudley, S. A. (2007). Above- and below-ground competition cues elicit independent responses. J. Ecol. 95, 261-272. doi: 10.1111/j.13652745.2007.01217.x

Murphy, G. P., and Dudley, S. A. (2009). Kin recognition: competition and cooperation in Impatiens (Balsaminaceae). Am. J. Bot. 96, 1990-1996. doi: 10.3732/ajb.0900006

O’Brien, E. E., Gersani, M., and Brown, J. S. (2005). Root proliferation and seed yield in response to spatial heterogeneity of below-ground competition. New Phytol. 168, 401-412. doi: 10.1111/j.1469-8137.2005.01520.x

Patti, G. J., Yanes, O., and Siuzdak, G. (2012). Innovation: metabolomics: the apogee of the omics trilogy. Nat. Rev. Mol. Cell Biol. 13, 263-269. doi: 10.1038/nrm3314

Peñuelas, J., Asensio, D., Tholl, D., Wenke, K., Rosenkranz, M., Piechulla, B., et al. (2014). Biogenic volatile emissions from the soil. Plant Cell Environ. 37, 1866-1891. doi: 10.1111/pce.12340

Pichersky, E., and Gershenzon, J. (2002). The formation and function of plant volatiles: perfumes for pollinator attraction and defense. Curr. Opin. Plant Biol. 5, 237-243. doi: 10.1016/S1369-5266(02)00251-250

Piechulla, B., and Degenhardt, J. (2014). The emerging importance of microbial volatile organic compounds. Plant Cell Environ. 37, 811-812. doi: $10.1111 /$ pce. 12254

Rasmann, S., Kollner, T. G., Degenhardt, J., Hiltpold, I., Toepfer, S., Kuhlmann, U., et al. (2005). Recruitment of entomopathogenic nematodes by insect-damaged maize roots. Nature 434, 732-737. doi: 10.1038/nature03451

Ryu, C.-M., Farag, M. A., Hu, C.-H., Reddy, M. S., Wei, H.-X., Paré, P. W., et al. (2003). Bacterial volatiles promote growth in Arabidopsis. Proc. Natl. Acad. Sci. U.S.A. 100, 4927-4932. doi: 10.1073/pnas.0730845100

Schloss, P. D., and Handelsman, J. (2004). Status of the Microbial Census. Microbiol. Mol. Biol. Rev. 68, 686-691. doi: 10.1128/MMBR.68.4.686-691.2004

Schmid, C., Bauer, S., Müller, B., and Bartelheimer, M. (2013a). Belowground neighbor perception in Arabidopsis thaliana studied by transcriptome analysis: roots of Hieracium pilosella cause biotic stress. Front. Plant Sci. 4:296. doi: $10.3389 /$ fpls.2013.00296
Schmid, C., Bauer, S., Müller, B., and Bartelheimer, M. (2013b). Belowground neighbor perception in Arabidopsis thaliana studied by transcriptome analysis: roots of Hieracium pilosella cause biotic stress. Funct. Plant Ecol. 4:296. doi: 10.3389/fpls.2013.00296

Schmidt, R., Cordovez, V., de Boer, W., Raaijmakers, J., and Garbeva, P. (2015). Volatile affairs in microbial interactions. ISME J. doi: 10.1038/ismej.2015.42

Schoenholtz, S. H., Miegroet, H. V., and Burger, J. A. (2000). A review of chemical and physical properties as indicators of forest soil quality: challenges and opportunities. For. Ecol. Manag. 138, 335-356. doi: 10.1016/S0378$1127(00) 00423-420$

Semchenko, M., Hutchings, M. J., and John, E. A. (2007). Challenging the tragedy of the commons in root competition: confounding effects of neighbour presence and substrate volume. J. Ecol. 95, 252-260. doi: 10.1111/j.13652745.2007.01210.x

Semchenko, M., Saar, S., and Lepik, A. (2014). Plant root exudates mediate neighbour recognition and trigger complex behavioural changes. New Phytol. 204, 631-637. doi: 10.1111/nph.12930

Sessitsch, A., Hardoim, P., Döring, J., Weilharter, A., Krause, A., Woyke, T., et al. (2012). Functional characteristics of an endophyte community colonizing rice roots as revealed by metagenomic analysis. Mol. Plant Microbe Interact. 25, 28-36. doi: 10.1094/MPMI-08-11-0204

Splivallo, R., Novero, M., Bertea, C., Bossi, S., and Bonfante, P. (2007). Truffle volatiles inhibit growth and induce an oxidative burst in Arabidopsis thaliana. New Phytol. 175, 417-424. doi: 10.1111/j.1469-8137.2007.02141.x

Steeghs, M., Bais, H. P., de Gouw, J., Goldan, P., Kuster, W., Northway, M., et al. (2004). Proton-transfer-reaction mass spectrometry as a new tool for real time analysis of root-secreted volatile organic compounds in Arabidopsis. Plant Physiol. 135, 47-58. doi: 10.1104/pp.104.038703

Wenke, K., Kai, M., and Piechulla, B. (2010). Belowground volatiles facilitate interactions between plant roots and soil organisms. Planta 231, 499-506. doi: 10.1007/s00425-009-1076-1072

Wenke, K., Weise, T., Warnke, R., Valverde, C., Wanke, D., Kai, M., et al. (2012). "Bacterial volatiles mediating information between bacteria and plants," in Biocommunication of Plants Signaling and Communication in Plants, eds G. Witzany and F. Baluška (Berlin: Springer), 327-347.

Wnuk, S., Kinastowski, S., and Kamiński, E. (1983). Synthesis and analysis of 1octen-3-ol, the main flavour component of mushrooms. Nahrung 27, 479-486. doi: 10.1002/food.19830270523

Conflict of Interest Statement: The authors declare that the research was conducted in the absence of any commercial or financial relationships that could be construed as a potential conflict of interest.

Copyright $\odot 2015$ Schenkel, Lemfack, Piechulla and Splivallo. This is an open-access article distributed under the terms of the Creative Commons Attribution License (CC BY). The use, distribution or reproduction in other forums is permitted, provided the original author(s) or licensor are credited and that the original publication in this journal is cited, in accordance with accepted academic practice. No use, distribution or reproduction is permitted which does not comply with these terms. 